\title{
Erratum to: Mathematical model of burden distribution for the bell-less top of a blast furnace
}

Zhao-jie Teng ${ }^{1)}$, Shu-sen Cheng ${ }^{1)}$, Peng-yu Du ${ }^{2)}$, and Xi-bin Guo ${ }^{3)}$

1) State Key Laboratory of Advanced Metallurgy, University of Science and Technology Beijing, Beijing 100083, China

2) MCC Capital Engineering and Research Incorporation Limited, Beijing 100176, China

3) Xuanhua Iron and Steel Company, Xuanhua 075100, China

Erratum to: International Journal of Minerals, Metallurgy and Materials

Volume 20, Number 7, July 2013, Page 620

DOI: $10.1007 / \mathrm{s} 12613-013-0775-7$

The original version of this article unfortunately contained a mistake. The presentation of Eq. (3) was incorrect. The correct version is given below:

$\frac{\mathrm{d}^{2} y}{\mathrm{~d} t^{2}}=-g \sin \alpha+\omega^{2}\left[z \sin \alpha \cos \alpha-(R-y) \cos ^{2} \alpha\right]-\eta \frac{\frac{\mathrm{d} y}{\mathrm{~d} t}}{m v} N$

The online version of the original article can be found at http://dx.doi.org/10.1007/s12613-013-0775-7

Corresponding author: Zhao-jie Teng E-mail: tengzhaojie2012@163.com

(C) University of Science and Technology Beijing and Springer-Verlag Berlin Heidelberg 2013 